\title{
The Relationship between Attachment, Depression, and Anxiety in Juveniles with Sexual Behavior Problems
}

\author{
Merino $\mathrm{C}^{1}$, Underwood LA ${ }^{2 *}$, Crump $\mathrm{Y}^{3}$ and Williams $\mathbf{C}^{2}$ \\ ${ }^{1}$ Department of Counselor Education, University of Wisconsin, Whitewater, USA \\ ${ }^{2}$ School of Psychology and Counseling, Regent University, Virginia, USA
}

${ }^{3}$ Louisiana Office of Juvenile Justice, Louisiana, USA

\begin{abstract}
The face of treatment for juvenile sex offending is changing, with a movement away from approaches based inforensic models toward more relational, holistic models. This movement has highlighted the importance of attachment experiences in the evaluation, assessment, and treatment of juveniles with sexual behavior problems (JSBPs) along with other important etiological factors, such as depression and anxiety. The current research utilized a correlational design to explore perceptions of attachment relationships, depression, and anxiety in a sample of incarcerated male JSBPs. Pearson product-moment correlation coefficients revealed several interesting relationships among the variables. By considering relationships between attachment and common etiological factors, clinicians may be better able to improve overall treatment outcome for youth and their communities.
\end{abstract}

Keywords: Juvenile with sexual behavior problem; Sex offenders; Depression; Anxiety

\section{Introduction}

The face of treatment for juvenile sex offending is changing. While treatment approaches based in forensic models have previously been the norm, more relational, holistic models of treatment are now emerging within the literature. This shift in focus has developed from the challenges of conducting empirical research with juveniles with sexual behavior problems (JSBPs) as well as the recognition that the literature related to evidence-based treatment approaches often contains contradictory findings This movement has also highlighted the importance of relational experiences, and specifically attachment experiences in the evaluation, assessment, and treatment of JSBPs. Just how attachment may play a role as a risk factor is still under debate. As part of a larger evaluation of treatment programs for JSBPs in the United States, the current research considers important correlations related to attachment and other etiological factors in a sample of male adolescents adjudicated for sexual offenses. Additionally, ways that a relational approach, and specifically an attachment framework, can be integrated into both treatment and research in order to "widen" the base of evidence in the treatment of juveniles who sexually offend is suggested. In fact, a more individualized approach based on attachment theory supports to critically consider attachment as an etiological factor for all JSBPs. In considering attachment as an etiological factor, clinicians may be better able to engage and work with youth within a relational framework and improve overall treatment outcome for youth and their communities.

\section{The Problem of Juvenile Sexual Offending}

The problem of juvenile sexual offending has been well-established. Adolescents (ages 12-18) commit approximately 20\% of rapes and anywhere from $20-50 \%$ of child sexual abuse cases in the United States each year $[1,2]$. Trends in rates of juvenile sexual offense arrests as well as recidivism over the last 10 years have shown little decline [3] JSBPs are overwhelmingly male, with only about $2-4 \%$ of adolescent sex offenders being female. In the United States, JSBPs are primarily Caucasian and come from two-parent homes. It is unknown if more JSBPs are Caucasian than other races, or if Caucasians tend to report more frequently [4]. Although there are higher numbers of juvenile
Caucasians charged with sex crimes each year, minority populations, such as African-Americans, Latinos, and other youth of color represent up to two-thirds of the incarcerated population [5]. Age can range from approximately 10 years of age to under 18 years with an average age of 14 for males and 13 for females [6].

Within the judicial system, sexual offending is defined as "non-consensual sexual behavior involving another person and encompassing force and/or manipulation" There are several different ways that JSBPs are classified in the literature, often based on either type of crime (rape or molestation) or type of victim (child or peer). Within the judicial system, there are several situations that can lead to charges of sexual offending. These include juveniles who may be engaged in consensual sexual activity, but based on legal standards are unable to consent. In these cases, both partners can be charged with a sexual offense. There is also the first time offender who has used force or manipulation with a victim. The victims can be much younger than the offender, or they can be peers or adults. Finally, there are repeat JSBPs who have already completed treatment and have continued to sexually offend. Among these are often those who are more aggressive or even violent in their assaults.

\section{Placement of JSBPs}

Based on pre-trial screening, assessment and judicial preference, an adolescent who has sexually offended may be placed in one of several different levels of treatment that reflect the risk of both reoffending (recidivism) as well as the needs of the juvenile [7]. The less restrictive placement available that balances both risk and the needs of the juvenile is recommended [8]. Offer a set of guidelines for making the decision

*Corresponding author: Underwood LA, School of Psychology and Counseling, Regent University, 1000 University Drive, CRB 215, Virginia Beach, Virginia 23464 USA, Tel: (757) 630-4442; E-mail: leeunde@regent.edu

Received December 20, 2017; Accepted December 27, 2017; Published January 05, 2018

Citation: Merino C, Underwood LA, Crump Y, Williams C (2017) The Relationship between Attachment, Depression, and Anxiety in Juveniles with Sexual Behavior Problems. J Ment Disord Treat 4: 152. doi:10.4172/2471-271X.1000152

Copyright: $\odot 2017$ Merino C, et al. This is an open-access article distributed under the terms of the Creative Commons Attribution License, which permits unrestricted use, distribution, and reproduction in any medium, provided the original author and source are credited. 
between residential and outpatient treatment settings. Incarceration is typically recommended for JSBPs in the following situations: (1) the offenses have been numerous and/or have involved more than one individual; (2) aggression was used during the assault(s); (3) severe emotional and behavioral problems are present; (4) antisocial attitudes are demonstrated; (5) there is poor motivation for treatment; (6) suicidal or homicidal ideation is present; (7) a volatile relationship at home threatens the safety of the individual; and finally (8) a victim is present in the juvenile's home. The juvenile's ability to acknowledge the offense and their willingness to engage in treatment are also important factors for determining the most appropriate level of care [9].

For offenders who are deemed lower risk, there are also community-based settings that provide treatment. Juveniles may begin treatment in a transitional facility; this level of care may be a structured residential treatment setting that focuses on both treatment and life skills. Group homes provide slightly less structure and provide more access to the community through school and work programs. Therapeutic foster care continues to be an option for some juvenile offenders who are transitioning into the community, where they live with foster families and may participate in day treatment or outpatient services. Community based programs can also be part of transition planning for reintegration into the community from a secure care setting [10]. Treatment approaches are often tailored to the needs of the particular setting, with those who are incarcerated receiving more intense, in-depth treatment while less intense forms are provided for those deemed lower risk of reoffending [11]. In considering a more relational treatment model, tailoring treatment to the setting would not be as important as assessing the needs of each individual youth.

\section{Widely Used Treatment Strategies}

There are several widely used treatment protocols for JSBPs. These models have met the definition of being "best practices." Within the juvenile justice system, evidenced-based treatments are defined as "a body of knowledge, also obtained through the scientific method, on the impact of specific practices on targeted outcomes for youth and their families" [12]. In working with juvenile corrections-based populations, evidenced-based treatments utilize several outcome principles. These include assessment of risks and needs, enhancing intrinsic motivation for change, providing objective interventions that are structured, skills training, using positive reinforcements, utilizing community resources for support, and providing measurable feedback through assessment of practices and processes.

There are several elements that appear to be core aspects of juvenile sex offender treatment and are often seen across evidenced-based protocols. The most common modalities for treatment include group and family interventions. Individual work is often not recommended as a primary modality for juvenile sex offending, but can be utilized to address other concerns, including psychopathology. Most treatments include psychoeducational component, given the developmental level and needs of juvenile populations. These are often delivered in a group or didactic format and are meant to enhance individual work. Treatment protocols include a variety of content, but most often are comprised of victim awareness, sex education, positive sexuality, anger management, social skills, cognitive restructuring, thinking errors, use of positive sexual images, assertiveness training, as well as elements of the sexual assault cycle.

Across disciplines, those who work with JSBPs recognize that there is no "one size fits all" approach to the treatment of JSBPs. In fact, "treatment success" is often defined as a reduction in the risk of sexually reoffending, also known as recidivism. From this forensic perspective, treatment is often linear and includes elements of psychoeducation as well as behavioral and cognitive behavioral (CBT) interventions targeting factors that might increase recidivism risk. While these approaches continue to have value and can be woven into a more relational approach to treatment, coming from a forensic focus only can be problematic For example, for many programs that work with youth who have sexually offended, public safety is a primary concern in placement and treatment decisions, as well as fulfilling the requirements of the legal and justice systems. Youth and their families become a secondary focus in the overall treatment process. However, satisfying legal and judicial requirements is a social priority in most parts of the world, and therefore should be part of the treatment focus. Finding a balance between forensic processes at one end and focusing completely on the juvenile and ignoring social consequences on the other continues to be an important task for clinicians. Additionally, understanding which risk factors to target in treatment requires careful attention to the etiology of sexual acting out for youth in treatment as well.

\section{Etiological Risk Factors}

There is much debate within the juvenile sexual offending literature with regards to factors that influence the development of sexually offending behaviors in juveniles. While research has highlighted possible factors that commonly contribute, clinicians realize that the development of sexually offending behaviors in youth is multidimensional, and unique to each individual. For example, cognitive dysfunction may be an important etiological factor for JSBPs [13]. Two main areas of cognitive impairment have also been identified, and include deficits with executive functions (planning, abstract thinking, impulse control, cognitive flexibility) and deficits in expressive and receptive language [14]. The "cycle of abuse" hypothesis is considered another important etiological factor in juvenile sexual offending. According to this hypothesis, JSBPs abuse because they have been sexually abused themselves. In fact, in a meta-analysis [15], JSBPs were five times more likely to have been sexually abused than their peers. Additional data suggest that anywhere from $32 \%-46 \%$ of juvenile sexual offenders report being sexually abused at some point prior to committing a sexual offense. Rates of physical abuse and neglect are also high. This becomes an important factor, as children who have been abused tend to show less empathy, struggle more with recognizing emotions in others, and have greater difficulty thinking abstractly.

Another etiological factor for juvenile sexual offending includes offender psychopathology. For example, depression has been extensively studied in the juvenile sex offender literature. Depressive symptoms in adolescence can be diagnosed as affective disorders, behavioral or emotional disorders specific to childhood, bipolar disorder, emerge from somatic conditions, or as posttraumatic stress disorder based on the context of the symptoms [16]. Presentation in adolescence can vary from that of adulthood, with anger or irritability being more prominent than the more typical melancholic type of depression [17]. Depression can affect youth in several ways, including clouding judgment and reason as well as affect sleep, energy levels and motivation in treatment [18]. Depressive symptoms in JSBPs have demonstrated stability across longitudinal studies, with younger adolescents experiencing higher levels of distress when compared to older adolescents [19]. Higher levels of depression are also linked to higher incidences of both sexual and non-sexual crimes, as well as greater levels of violence associated with offending [20].

Anxiety has also been proposed as an etiological factor in juvenile 
sexual offending. Anxiety can take many forms, including more generalized anxiety, obsessive-compulsive tendencies, panic disorder and phobias. Anxiety disorders affect youth in several key ways. For example, they may experience nervousness, apprehension, fear, and tension regularly. This can lead to problems with concentration and routine tasks. Symptoms of panic are not uncommon, and the unpredictable nature of the symptoms can be debilitating. Youth who have experiences past trauma may also experience intrusive memories that can heighten anxiety JSBPs may also demonstrate higher levels of anxiety in social situations, making peer and adult relationships more difficult Suicidal tendencies may also be elevated among JSBPs. There do not appear to be, however, higher incidences of psychotic or other dissociative symptoms. Conduct problems are frequently seen in JSBPs, along with antisocial tendencies. In terms of personality characteristics, dependent, schizoid, and avoidant traits are found most often in this population. Because of the prevalence of mental health needs of JSBPs, most treatment approaches assess for and may intervene with pharmacotherapy as needed to address these concerns.

Relationships can also be crucial in the development of sexually offending behaviors in numerous ways. For example, a higher rate of family dysfunction has been found among JSBPs. Types of dysfunction include parental rejection and/or separation, physical abuse and/or neglect, familial substance abuse, and a pattern of criminal behavior within the family Adolescent sex offenders also tend to struggle specifically with peer relationships and may lack developmentally appropriate social skills. For example, within social relationships many JSBPs have difficulty initiating, engaging in conversation, and decoding affective and other social cues during peer interactions. Some JSBPs report feelings of loneliness, low self-esteem, and report isolating themselves from peers as a result of difficulties in peer relationships. There is also frequently a preoccupation with the self and excessive selfabsorption. Because relational factors are prevalent when considering treatment of JSBPs, treatment approaches that focus on relationships may ultimately be preferred to those that focus more on recidivism or other factors. This highlights the importance of attachment theory, and attachment style as a possible etiological factor for JSBPs.

\section{Attachment Theory}

Attachment theory was first proposed by John Bowlby as an "innate biological system" found in humans with behaviors that seek protection and comfort from a primary caregiver in times of stress. Attachment and sexual offending was first considered in adult male population studies focusing on social relatedness and empathy. The common consensus in the field of sexual offender treatment is that attachment differences do exist in sex offenders as compared to other types of offenders and that they are significantly linked to the development of sexually abusive behavior in males. However, the role of attachment has yet to be significantly linked causally through research. In current theory, attachment is at once process, a set of organized procedures, and a state of being. Attachment theory utilizes a developmental lens to view relational problems, and initially was designed to explain behaviors and intervene in childhood to ensure optimal outcome [21]. The theory has, however, become influential not only in the clinical treatment of children, but also for treatment across the lifespan [22]. Attachment experiences in childhood influence all later relationships in some way, and can account for difficulties in psychological and relational functioning in adolescence and adulthood [23]. As a result attachment theory has become an important etiological factor for explaining and intervening with JSBPs [24,25]. The work of John and his protégé Mary Ainsworth focused primarily on describing and categorizing attachment in infancy. Behaviors that draw the caregiver to the infant serve to express and meet needs and also to regulate internal states. The primary caregiver's response to the infant's behaviors results in the creation of an internalized object known as the internal working model. The internal working model contains affective triggers that stimulate behaviors from the infant and in turn influence responses of the caregiver. The internal working model has been described as the "container, assimilator, and synthesizer of experience". Internal working models cannot be directly observed, but can be studied based on the behaviors exhibited by the infant. Mary Ainsworth and her colleagues further explored the behavioral outcomes of internal working models in infants through the development and use of the Strange Situation. She observed that infants responded to behaviors from their caregivers in distinct ways and created a system for naming and defining styles of attachment. She and her colleagues found that various categorizations of attachment style influenced behaviors and relationships into adulthood. Ideally, infants develop secure attachment, trusting in their caregivers to provide for their needs. Other, more problematic styles include anxious, avoidant, and disorganized. Although both anxious and avoidant styles can lead to significant problems later in life, they are not considered maladaptive per se, only suboptimal. Disorganized attachment carries the greatest risk of psychopathology and is considered maladaptive.

Attachment theory as defined in infancy is often inadequate when considering the developmental changes that occur as a child matures. Adolescence is a time when most relationships require reorganization. Many adolescents begin to rely less on their parents emotionally and begin to form closer and deeper relationships with peers [26,27]. These friendships form the basis of later, reciprocal attachment relationships in adulthood. Although there is less reliance on parents to meet emotional needs, adolescents will still turn to their parents in times of stress. Attachment to parents also influences the quality of new peer relationships [28]. Secure attachment in adolescence is associated with a variety of positive cognitive, emotional, social, and behavioral outcomes. Secure adolescents are often more socially competent, possessing advanced emotional skills such as empathy, the ability to express affect, and an awareness of the feelings of others. They also tend to display higher levels of identity development, which is a critical task in adolescence. Having a strong sense of identity has been associated with a greater ability to form meaningful intimate relationships. Securely attached adolescents also demonstrate better coping skills than their insecurely attached peers.

Insecure attachment in adolescence has been associated with numerous maladaptive outcomes across life domains. Insecurely attached adolescents tend to use more avoidant coping strategies when faced with new environmental situations or in their relationships with parents [29]. Many adolescents with poor attachment become very preoccupied with relationships and demonstrate discomfort in social situations. This significantly raises the risk of depression and anxiety disorders as well as substance use and suicidal risk. Insecurely attached adolescents may also view most relationships as untrustworthy and are more likely to assume hostility in unknown situations. This can likewise raise the risk of externalizing behaviors. Insecure attachment styles may also be reinforced by ongoing problematic relationships with parents and siblings as well as hostile environmental factors. These difficult life circumstances can also make it difficult for the adolescent to create more meaningful relationships with peers or potential romantic partners. For insecurely attached adolescents, their internal working models generalize many negative experiences and interactions from the past to current situations, creating difficulties in functioning [30]. 
Assessing attachment styles in adolescence is less precise than in infancy. For infants, attachment can be directly observed through behaviors. While there are behavioral manifestations for adolescents and adults, attachment often needs to be examined at a more representational level. To evaluate attachment at this level, the Child Attachment Interview was created by modifying the Adult Attachment Interview used with adults. The CAI, however, was intended for children ages 8-12, and has occasionally been utilized for adolescents up to age 16. It also requires a good deal of training, supervision, and is time consuming. A self-response instrument, the Adolescent Attachment Questionnaire [31] was developed specifically for adolescents from 12-19 years. The AAQ focuses on the three dimensions of attachment perceptions of anger/distress, availability, and goal-corrected partnership. The AAQ can provide needed information regarding individual attachment styles in adolescence, allowing treatment providers and researchers alike to assess and utilize attachment in examining etiological risk factors for sexual offending.

\section{Attachment Theory and Sexual Offending}

Research specific to the relationship between attachment and sexual offending is not as clear. One proposed link between attachment style and sexual offending in adults occurs as a result of experiencing an overlap between the attachment and sexual systems beginning in childhood and early adolescence. The development of sexuality during adolescence and adulthood is conceptually separate from the attachment. However, the two constructs are strongly interconnected, in that secure attachment strongly predicts psycho-sexual maturity and adjustment in adulthood. Insecurely attached adolescents have been shown to use sex more frequently to deal with conflicts about their ability to develop intimacy with others. Additionally, regardless of physical satisfaction derived from sexual acts, insecurely attached adolescents who utilize sex in this way remain emotionally isolated. Based on work with adult sex offenders, presents a model of sex offender types based on the attachment and Crittenden. He proposes that preoccupied attachment leads to a propensity for child molestation, where the offender holds a negative view of self and an insecure view of others. Dismissive attachment leads to a propensity for rape of adult victims, and is characterized by a positive view of the self and a negative view of others. Fearful attachment is connected with sexually offending against child victims and is associated with a negative view of self and others also suggests that this typology may be true for JSBPs as well, and calls for further research of attachment profiles and sex offender typology.

The most comprehensive theory of attachment and sexual offending, which emphasizes that an insecure attachment style can render an individual vulnerable to sexual offending. Attachment is not the cause of sexual offending per se, but interacts with other predisposing and precipitating factors that can lead to sexual abuse. A model [32] also emphasizes the use of sexualized coping, such as excessive masturbation, pornography, and sexual acts with others, as a way to avoid difficult family and environmental factors. Sexually coercive behaviors develop as a result and become conditioned responses, maintained by cognitive distortions. Marshall's model has also been built upon by other researchers, including sexual fantasies as a mode of sexualized coping. Studies [33] further indicate that attachment in childhood is often directly related to the development of adolescent sexual attitudes and behavioral patterns. For youth with insecure or disorganized attachments, sexual relationships are often a means for gaining control and meeting personal needs, rather than for mutual emotional and physical satisfaction.

\section{Current Study}

Research on attachment theory and juvenile sexual offending is still in its infancy. However, strides have been made to integrate attachment patterns as part of a larger, relational approach to treatment. There are many strengths in doing this clinically. For example, approaching treatment with youth who have sexually offended from a place of empathy, recognition of their humanity, and a focus on improving their relationships with others is clinically different than approaching from a place of punishment and judgement for past behaviors. Additionally, youth who are supported and helped from a more relational approach may increase their investment in treatment. While this remains speculation only, further research is needed. There are several challenges related to ongoing clinical research with JSBPs. For example, it is often not ethical to withhold treatment to create a control group for comparative studies. Youth are also considered vulnerable in many ways, and gaining access to them can be another challenge. This may result in less statistical power due to smaller population sizes. It becomes imperative for clinicians and others in the field to recognize patterns and trends from research despite these flaws, and continue to replicate and stretch the field in new directions.

The current study aims to present correlational statistics related to perceptions of attachment relationships with primary caregivers from a group of incarcerated youth who have sexually offended. Data was collected as part of a larger program evaluation piloting a new approach to treatment detailed elsewhere [34]. Interestingly, correlational data highlighted several important relationships between perceptions of attachment relationship and other etiological factors, including depression and anxiety. A descriptive correlational approach was then utilized to further examine the available data. The principle research question driving this analysis was to provide a description of the sample of youth who have sexually offended in terms of attachment perceptions and other important etiological factors, including depression and anxiety. By learning more about the relationships between these variables, contributions to the growing literature regarding an attachment-informed relational treatment approach for this population can be made.

\section{Methods}

\section{Participants}

Participants for the larger study were recruited from youth in correctional facilities for sexual offenses in both the Northwest and Southeastern regions of the United States. A total of 51 male juveniles participated in the study. The age range of participants was $11.5-20$ years, with a mean age of 15.6 years. In terms of ethnicity, $23.5 \%(n=12)$ reported being Caucasian, $29.45(\mathrm{n}=15)$ African American, 3.9\% $(\mathrm{n}=2)$, Hispanic, $7.8 \%(n=4)$ Native American, and $21.6 \%(n=11)$ as multiethnic. A Human Services Review Committee reviewed and approved the original study based on safeguards and human protection factors. Potential participants and their legal guardians were informed of the duration of the study, the nature of treatment options, the instruments used in pre- and post- testing, and the purposes and design of the study.

\section{Instrumentation}

Data from the following instruments was analyzed in the current study.

\section{Demographic questionnaire}

Participants were asked to fill out a demographic questionnaire 
upon agreeing to participate in the research. The demographic questionnaire obtained information on age, race/ethnicity, level of education completed, past history of sexual abuse received, past history of charged sex offense, and past history of confinement and treatment.

\section{The adolescent attachment questionnaire}

The AAQ is a brief self-report instrument for adolescents from ages 12-20. The AAQ consists of nine questions that are rated on a five-point Likert scale and assesses the adolescent's perceptions of the availability and responsiveness of their caregiver. The AAQ is based on dimensions which are specific to defining parent-adolescent attachment. The AAQ does not provide a total score, rather focuses on the three dimensions of attachment perceptions of anger/distress, availability, and goal- corrected partnership. Each of the scales have three items, and scores range from 3-15. The Anger/Distress Scale measures negative affective responses to the perceived unavailability of the attachment figure. The Availability scale assesses the adolescent's confidence in the availability and responsiveness of the attachment figure. Goal-corrected Partnership assesses the extent to which the adolescent thinks about and is empathic to the needs of their attachment figure. The AAQ has demonstrated good divergent validity with the Adolescent Attachment Interview although it does not categorize youth as "secure" or "insecure." The reliability of the AAQ has been demonstrated as acceptable, with alpha coefficients ranging from 0.62 to 0.80 . Test-retest reliability with normative samples ranged from 0.68 to 0.74 , indicating temporal stability The AAQ is administered in paper and pencil format.

\section{Children's depression inventory-2}

The CDI-2 is a self-report instrument for children ages 7-17. The CDI-2 relies on self-reporting by children and adolescents, and also includes versions for teachers and caregivers. The inventory can be administered and scored in a paper and pencil format or online. In the current study, only the self-report form for children was utilized. The instrument asks youth to consider their symptoms over the past two weeks. The inventory identifies several key depressive risk factors, including emotional problems, functional problems, negative mood, negative self-esteem, ineffectiveness, and interpersonal problems. Reliability measures for the CDI-2 range from 0.75-0.91, indicating moderately high to high levels of consistency in identifying depressive symptoms. Internal consistency in the literature ranges from 0.790.89 , and test-retest reliability following $2-4$ weeks ranges from 0.92 0.98 . Discriminant validity has been demonstrated at approximately $80 \%$, indicating a strong ability to identify those children who have depressive symptoms from those who do not

\section{Revised children's manifest anxiety scale (RCMAS-2; Reynolds and Richmond)}

The RCMAS-2 is a self-reporting instrument designed to gather and rate the nature of anxiety experienced by children and adolescents aged 6-19 years. Questions are posed as "yes/no" items. A "yes" response indicates that the item in question describes the respondents feeling and actions. A "no" response indicates that the item is generally not descriptive of the respondents feelings and actions. A total anxiety score is calculated on the basis of 28 items divided into three subscales measuring (1) physiological anxiety (such as sleep difficulties, fatigue, and nausea), (2) worry and over-sensitivity (such as obsessive concerns about having one's feelings hurt or feeling emotionally isolated), and (3) social concerns related to interpersonal relations and levels of concentration. The scale also contains a "Lie" subscale assessing the respondent's level of lie or anxiety on that subscale. Since the scales are self-reported, the lie subscale is used to detect respondents who are "faking" being good by answering in the affirmative to questions they know would reflect upon them positively.

Several studies have established the reliability of RCMAS as a self-reporting instrument to measure for child anxiety and predicted relationships between anxiety, depression, PTSD, delinquency, age, sex, and somaticism. Psychometric evaluations have also established reliability of the CMAS in American, German, Spanish, and FrenchCanadian populations. Evidence for the construct validity of the RCMAS has been obtained in numerous studies based on observed correlations predicted between anxiety, depression, PTSD, delinquency, age, and sex).

\section{Procedures}

The current study utilizes archival data gathered during a larger study of JSBPs as reported elsewhere. All archival data resulted from data collection at both sites lead by the principal investigator (PI). In order to ensure confidentiality of data, the current researcher received all data directly from the PI. No names are associated with any of the data that was utilized. Only the PI had access to the master list which contains names. At no time was this list accessed by the current investigator. Following Human Subjects Review Committee approval for the current study, archival data was provided to the current investigator for analysis in spreadsheet format. Data was then uploaded and analyzed in SPSS.

\section{Results}

\section{Characteristics of the sample}

As previously reported the total size of the sample was 51 males with an average age of 15.6 years. $45.1 \%(n=23)$ reported having received prior treatment for sexual offenses before their current placement. In terms of time in their current placement, $31.4 \%(n=16)$ indicated a stay of at least nine months or more. In terms of past victimization, $62.7 \%$ $(n=32)$ indicated that they had been abused sexually. For the current study, $64.7 \%$ of youth identified their primary caregiver as their mother $(\mathrm{n}=33)$. A summary is presented in Table 1.

Descriptive statistics related to the three scales of the AAQ also provide information related to perceptions of attachment relationships with primary caregivers for this sample. For instance, the Anger/ Distress Scale measures negative affective responses to the perceived unavailability of the attachment figure, with higher scores indicating greater anger and distress toward the primary caregiver. Youth in the current study identified moderate levels of anger toward their primary caregivers. The Availability scale assesses the adolescent's confidence in the availability and responsiveness of the attachment figure, with higher scores indicating greater confidence. Overall the youth in the study felt confident in the availability of their caregivers. Finally Goalcorrected Partnership assesses the extent to which the adolescent thinks about and is empathic to the needs of their attachment figure, with higher scores indicating the ability to empathize with their caregivers. Again, this sample appeared able to think about the needs of their caregivers separate from their own as well as feel empathy for them. Table 2 provides the means and standard deviations for the AAQ scales Depression was measured using the CDI-2. Higher scores indicate a higher level of distressful symptoms related to depression. The mean score for the current sample was average and did not indicate significant levels of depression-related symptoms. In terms of anxiety, the mean score was also average for the group, indicating an average 


\begin{tabular}{|c|c|c|}
\hline Characteristic & $\mathbf{n}$ & $\%$ \\
\hline \multicolumn{3}{|l|}{ Age } \\
\hline $11-13$ years & 9 & 17.7 \\
\hline $14-16$ years & 24 & 47.1 \\
\hline $17-20$ years & 18 & 35.3 \\
\hline \multicolumn{3}{|l|}{ Ethnicity } \\
\hline Caucasian & 12 & 23.5 \\
\hline African American & 15 & 29.4 \\
\hline Hispanic American & 2 & 3.9 \\
\hline Native American & 4 & 7.8 \\
\hline Multi-Ethnic & 11 & 21.6 \\
\hline Other & 7 & 13.7 \\
\hline \multicolumn{3}{|c|}{ Prior Treatment for Previous Sexual Offenses } \\
\hline Yes & 23 & 45.1 \\
\hline No & 24 & 47.1 \\
\hline \multicolumn{3}{|c|}{ Time in Current Treatment Facility } \\
\hline $0-3$ Months & 4 & 7.8 \\
\hline 4-6 Months & 9 & 17.6 \\
\hline 7-9 Months & 7 & 13.7 \\
\hline 9-12 Months & 16 & 31.4 \\
\hline $12+$ Months & 15 & 29.4 \\
\hline \multicolumn{3}{|c|}{ Victim of Sexual Abuse } \\
\hline Yes & 32 & 62.7 \\
\hline No & 19 & 37.3 \\
\hline \multicolumn{3}{|l|}{ Primary Caregiver } \\
\hline Mother & 33 & 64.7 \\
\hline Father & 11 & 21.5 \\
\hline Grandparent & 5 & 10 \\
\hline Foster Parent & 2 & 3.8 \\
\hline
\end{tabular}

Table 1: Demographic characteristics of participants $(\mathrm{N}=51)$.

\begin{tabular}{|c|c|c|}
\hline Measure & M & SD \\
\hline AAQ-Angry/Distress & 6.14 & 3.11 \\
\hline AAQ-Availability & 11.65 & 3.34 \\
\hline AAQ-Goal Directed & 13.08 & 2.72 \\
\hline CDI-2 Total Score & 54.98 & 11.04 \\
\hline RCMAS Defensiveness & 48.11 & 10.15 \\
\hline RCMAS Total Score & 51.35 & 11.83 \\
\hline RCMAS-Physical & 48.49 & 10.92 \\
\hline RCMAS-Worry & 52.74 & 11.62 \\
\hline RCMAS-Social Anxiety & 51.39 & 12.04 \\
\hline
\end{tabular}

Table 2: Means and standard deviations for measures of etiological factors.

level of symptoms of anxiety. The Defensiveness scale for the sample on the RCMAS was also average $(\mathrm{M}=48.11, \mathrm{SD}=10.15)$, indicating that the results are within the acceptable range for interpretation. The RCMAS also provides several additional scores that provide additional information about the types of symptoms the youth might be experiencing. Of the three subscales, Worry $(\mathrm{M}=52.74, \mathrm{SD}=11.62)$ was slightly higher. Physical Symptoms $(\mathrm{M}=48.49, \mathrm{SD}=10.92)$ and Social Anxiety $(\mathrm{M}=51.39, \mathrm{SD}=12.04)$ were somewhat lower. However, all three scales provide information related to anxiety for the sample, in that items related to general worry were somewhat more prevalent, followed by social anxiety and then physical anxiety symptoms. See Table 2 for a summary of means and standard deviations.

\section{Intercorrelations between etiological factors}

To explore relationships among the variables, Pearson productmoment correlation coefficients were computed. Preliminary analyses were performed to ensure no violation of the assumptions of normality, linearity and homoscedasticity. Intercorrelations are reported in and summarized here. When considering perceptions of attachment relationships with primary caregiver for the entire sample, several significant relationships emerged. First, the AAQ Angry/Distress scale and the AAQ Availability scales were negatively correlated at the $\mathrm{p}<.001$ level. In other words, as anger and distress in the relationship with primary caregiver increases, perceptions of caregiver availability decrease. This correlational relationship, based on Cohen's (1988) interpretation model, is moderate, sharing only $14.44 \%$ of the variance. $\left(r^{2}=0.1444\right)$. The AAQ Goal Directed Partnership scale was also correlated with the AAQ Availability scale in a positive direction- meaning that as the ability to think about the parent as well as feel empathy toward them increases, the perceptions of caregiver availability also increased. The strength of this relationship is large, accounting for $50 \%$ of the variance $\left(r^{2}=0.5041\right)$ [35].

Perceptions of attachment relationships with caregiver had several significant relationships with depression score as measured by the CDI-2. The AAQ Angry/Distress scale was positively correlated with depression score, indicating that, as anger and distress escalate within the relationship with their caregiver, depression related symptoms tended to increase as well. While statistically significant, this relationship is considered moderate, accounting for only $15 \%$ of the variance $\left(\mathrm{r}^{2}=0.1521\right)$. The AAQ Availability scale and depression score had a significant negative relationship. Also moderate in strength $\left(\mathrm{r}^{2}=0.1296\right)$, this finding suggests that as depression score increases, perceptions related to caregiver availability decrease. While only about $13 \%$ of the variance is shared in this correlation, relationships between attachment and depression may be an important issue clinically. Interestingly, perceptions of attachment relationships with primary caregiver as measured by all three AAQ scales were not significantly correlated with anxiety as measured by the RCMAS.

Several other significant relationships between depression and anxiety also emerged. Total depression score on the CDI was moderately correlated with total anxiety score, physical symptoms, and worry as measured by the RCMAS. Moderate negative correlations were also found between the RCMAS Defensiveness scale and the Physical, Worry, Social Anxiety, and Total scales. While this is likely an expected relationship, it is important to note that, as defensiveness increased, anxiety scores decreased. Large correlations were identified between scales and the total RCMAS score, a finding that is expected due to the nature of the measure. Table 3 summarizes the correlation coefficients for these scales.

\section{Discussion}

The current study provides an exploration of attachment with a small group of incarcerated youth who have sexually offended. This exploration has highlighted several important relationships between other etiological factors, specifically depression and anxiety, and the perceptions of attachment relationship with primary caregivers. The AAQ has been in use for several decades as a way of measuring perceptions of attachment. These perceptions, in turn, can provide information related to possible attachment style, upon which most attachment-based treatment approaches are based. The AAQ is a short, relatively simple instrument to administer and score, and with continued research, may emerge as a mainstay in the assessment of attachment in adolescence. To our knowledge, the AAQ has not been widely utilized to assess attachment perceptions in male JSBPs, and before further research can be designed, the question of whether or not there are significant relationships between attachment and other exploratory factors needed to be addressed. The current study was implemented with this in mind. 


\begin{tabular}{|c|c|c|c|c|c|c|c|c|c|}
\hline Measure & 1 & 2 & 3 & 4 & 5 & 6 & 7 & 8 & 9 \\
\hline AAQ-Angry/Distress & ---- & & & & & & & & \\
\hline AAQ-Availability & $-0.38^{* *}$ & --- & & & & & & & \\
\hline AAQ-Goal Directed & -0.25 & $0.71^{\star *}$ & --- & & & & & & \\
\hline CDI-2 Total Score & $0.39^{\star *}$ & $-0.36^{\star *}$ & -0.07 & ---- & & & & & \\
\hline RCMAS Defensiveness & -0.06 & -0.03 & -0.05 & -0.09 & --- & & & & \\
\hline RCMAS Total Score & 0.09 & -0.02 & -0.02 & $0.37^{* *}$ & $-0.45^{\star \star}$ & --- & & & \\
\hline RCMAS-Physical & 0.15 & 0.08 & 0.01 & $0.46^{\star *}$ & $-0.45^{\star \star}$ & $0.78^{\star *}$ & ---- & & \\
\hline RCMAS-Worry & 0.10 & -0.10 & -0.03 & $0.32^{*}$ & $-0.37^{* *}$ & $0.94^{* *}$ & $0.61^{*}$ & --- & \\
\hline RCMAS-Social Anxiety & -0.02 & 0.01 & -0.05 & 0.20 & $-0.36^{* *}$ & $0.88^{* *}$ & $0.54^{* *}$ & $0.77^{\star *}$ & --- \\
\hline
\end{tabular}

Note: $\mathrm{N}=51$. ${ }^{*} \mathrm{p}<0.05$ (two-tailed); ${ }^{* *} \mathrm{p}<0.001$ (two-tailed). $\mathrm{AAQ}=$ Adolescent Attachment Questionnaire; CDI=Children's Depression Inventory; RCMAS=Revised Children's Manifest Anxiety Scale.

Table 3: Intercorrelations among measures of etiological factors.

\section{Perceptions of attachment}

Research on attachment theory continues to highlight the importance of relationships across the lifespan. For adolescents, attachment relationships begin to shift, with parents taking on a more secondary role. However, adolescents continue to turn to caregivers in times of stress, and their perceptions of their relationship with their primary caregiver continues to serve important functions. For the current sample of juvenile males, perceptions of caregiver attachment were overall positive as measured by the AAQ. This is an important finding, as many of these youth have been away from their primary caregivers for extended lengths of time. Despite being incarcerated, youth in the current study continued to feel that their caregiver was available to them, felt only moderate amounts of anger toward their caregiver, and demonstrated a moderate ability to feel empathy for and perceive the needs of their caregiver.

The correlations between scales on the AAQ also provide new information related to attachment and this sample. The negative relationship between the Angry/Distress scale and the Availability scale is not surprising, given that greater anger toward an attachment figure would likely affect perceptions of their availability to help when needed. Likewise, the interrelationship between the Goal-Directed Partnership scale and the Availability scale is also intuitive. When an adolescent feels that their parent is available, they are likely better able to work together toward common goals. They are also more likely to carry an overall positive impression of their parent with them. When considering treatment from an attachment perspective, the presence of moderately positive perceptions of relationships could be a strength upon which the clinician can build. In fact, an attachment-informed treatment approach with JSBPs provides a way for the therapist to consider such attachment themes and how they may be expressed, either consciously or unconsciously, by the adolescent. Assessing each juvenile and examining their AAQ scores for patterns can help in case conceptualization from an attachment-informed approach, aiding in interventions that can provide corrective relational experiences for youth who struggle in their relationships, or provide additional positive growth for youth with positive attachment perceptions.

\section{Depression and attachment}

Symptoms of depression, as measured by the CDI-2, were low to moderate in the current sample. This reflects previous findings in the literature related to depression and male JSBPs. Level of depression was correlated with both the AAQ Availability scale as well as the AAQ Angry/Distress scale. While only moderate in strength, these relationships provide additional information related to patterns for clinicians working with this population. First suggested that attachment style, particularly anxious and avoidant patterns, may be significant risk factors for the development of depression in adolescence. For youth in the current study with higher depression scores, there appears to be a greater possibility of attachment problems with primary caregivers. Depression along with other mood disorders is often carefully screened for during assessment so that depression-specific treatment can be implemented. The possibility that attachment difficulties could also be present in youth with higher depression scores contributes to a more thorough assessment and aides in treatment planning. Also, armed with information related to perceptions of attachment relationships, perhaps approaching treatment depression in male adolescents who have sexually offended from a more relational perspective could be effective in managing and treating symptoms of depression.

\section{Attachment and anxiety}

Interestingly, there were no significant relationships between the three attachment scales of the AAQ and the scales of the RCMAS. In adolescence, generalized anxiety disorder is the most commonly diagnosed anxiety disorder. Generalized anxiety disorder symptoms in adolescence have been correlated with perceived attachment difficulties with primary caregivers, such as a sense of alienation, lower levels of trust, and poor communication. The current sample had a low to moderate total anxiety score, and likely most would not meet criteria for an anxiety disorder diagnosis, such as generalized anxiety disorder. This may partially explain the lack of significant relationships between attachment and anxiety. A larger sample and continued research might lead to a more significant relationship between these factors. While not yet significantly related, the relationships between anxiety and depression may be of interest, given the correlations between attachment and depression. Again, further research could examine these factors to determine what, if any, relationships may exist.

\section{Treatment applications}

The premise guiding the current study is to present attachment as a viable etiological factor worth considering in the assessment and treatment of juvenile sexual offending, along with others such as depression and anxiety. Several statistically significant relationships among these variables emerged in the sample. Beyond statistical significance, the issue of clinical utility is also important to address. In suggesting that attachment is an important construct in working with male JSBPs, a way to then integrate the information into attachmentinformed treatment becomes an important function. Attachment theory is largely developmental, and does not include specific interventions or techniques that are utilized in clinical work. An attachment framework is, however, a way to approach thinking about the client and focusing on relational experiences. In providing additional 
Citation: Merino C, Underwood LA, Crump Y, Williams C (2018) The Relationship between Attachment, Depression, and Anxiety in Juveniles with Sexual Behavior Problems. J Ment Disord Treat 4: 152. doi:10.4172/2471-271X.1000152

Page 8 of 9

information regarding relationships between attachment and other variables, the current study joins a growing body of attachment related literature for juvenile sex offender treatment. We echo the approaches suggested by in approaching juveniles from an attachment perspective. We also recognize the need to dramatically shift current treatment approaches in order to centralize the importance of relationships. It is not uncommon in the literature regarding treatment outcomes to find the therapeutic relationship as one of the most influential factors in treatment success. Attachment-informed practice already approaches individual clients from a relational place, without harsh judgement, and with a willingness to individualize treatment instead of adhering to strict protocols. Current findings highlight the relationships of attachment to other important etiological factors that are often a focus on more traditional treatment approaches to juvenile sex offending.

\section{Limitations of the Current Study}

There are several limitations worth noting in the current research. This study was designed as a preliminary exploration examining attachment relationships in a sample of male JSBPs in incarceration. Because the study is largely descriptive, only a sense of interrelationships among these constructs can be provided. Because correlational research cannot indicate causation, further replication of the current research is needed. Additionally, the sample size was small due to difficulties in accessing this vulnerable population as well as obtaining informed consent from multiple parties. Continuing this research with multiple groups becomes important. Instruments were also self-report, and are therefore subjective to the adolescents' experiences. Bias, error, and individual differences may have influenced the data. The AAQ was designed to assess attachment perceptions in adolescence, yet has not been widely utilized, particularly with youth in corrections who have sexually offended. There may or may not be some limitations as a result of using a new instrument with the current population.

\section{Suggestions for Further Research}

Throughout the current study we have highlighted the need to continue this line of research based on the call by, who suggested that attachment be assessed for all JSBPs in order to address relationship needs within treatment. Most importantly, the current findings suggest that relationships between attachment and other etiological factors, specifically depression and anxiety do exist on a small scale, and likely can continue to be explored with larger and more diverse sample sizes. Utilizing the AAQ as a simple and effective way to assess attachment is also presented in the current study and requires additional research to confirm its utility. Continuing to identify ways of improving outcomes for juvenile sex offender treatment is an important task. Building on the current study can continue to contribute to the literature and generate new applications to theory and treatment of male juveniles who have sexually offended.

\section{Conclusions}

In considering treatment and rehabilitation for JSBPs, the movement toward more relational approaches is likely to continue. In working with JSBPs, the need to consider the interrelationships among factors that can place youth at higher risk and tailor treatment appropriately is an important task. The current study contributes to this growing literature through the exploration of attachment as an etiological factor and its relationships to depression and anxiety. The use of the AAQ successfully with this population is a milestone, and a better understanding of how perceptions of attachment relationships and mental health issues interact provides a promising direction for the future of treatment with male juveniles with sexual behavior problems.

\section{References}

1. Rich P (2011) Understanding, Assessing, and Rehabilitating JSBPs

2. Timothy K (2012) The Internal World of the Juvenile Sex Offender: Through a Glass Darkly Then Face to Face.

3. Underwood LA, von Dresner KS, Phillips AL (2006) Community treatment programs for juveniles: A best-evidence summary. International Journal of Behavioral and Consultation Therapy 2: 286-304.

4. Robertiello G, Terry K (2007) Can we profile sex offenders? A review of sex offender typologies Aggression and Violent Behavior 12: 508-518.

5. Underwood LA, Phillips A, von Dresner K, Knight PD (2006) Critical factors in mental health programming for juveniles in corrections facilities. International Journal of Behavioral and Consultation Therapy 2: 108-141.

6. Bourke ML, Donohue B (1996) Assessment and treatment of JSBPs: An empirical review. Journal of Child Abuse 5: 47-70.

7. Stops M, Mays GL (1991) Treating adolescent sex offenders in a multi-cultural community setting. Journal of Offender Rehabilitation 17: 87-103.

8. Underwood L, Washington A (2016) Mental Illness and Juvenile Offenders. International Journal of Environmental Research and Public Health 13: 1-14.

9. Ward T (2014) The explanation of sexual offending: From single factor theories to integrative pluralism. Journal of Sexual Aggression 20: 130-141.

10. Underwood LA, Knight $P$ (2006) Treatment and postrelease rehabilitative programs for juvenile offenders. Child and Adolescent Psychiatric Clinics of North America 15: 539-556.

11. Underwood LA, Robinson SB, Mosholder E, Warren KM (2008) Sex offender care for adolescents in secure care: Critical factors and counseling strategies. Clinical Psychology Review 28: 917-932.

12. Righthand S, Welch C (2004) Characteristics of JSBPs. Journal of Child Sexual Abuse 15-32.

13. Seto MC, Lalumiere ML (2010) What is so special about male adolescent sex offending.

14. Bomba J, Modrzejewska R (2008) Prospective study on dynamics of depression in mid-adolescence Archives of Psychiatry and Psychotherapy 1: 29-35.

15. Ritakallio M (2006) Delinquency and the profile of offences among depressed and non-depressed adolescents. Criminal Behaviour and Mental Health 16 100-110.

16. Underwood LA, Warren KM, Talbott L, Jackson L, Dailey FL (2014) Menta health treatment in juvenile justice secure care facilities: Practice and policy recommendations. Journal of Forensic Psychology Practice 14: 55-85

17. Yaqoob N, Kha MA (2014) Patterns of depression, anxiety symptoms, and coping styles among early and late adolescent students. Pakistan Armed Forces Medical Journal 64: 573-576.

18. Imbach D, Aebi M, Metzke C, Bessler C, Steinhause H (2013) Internalizing and externalizin problems, depression, and self-esteem in non-detained male juvenile offenders. Child and Adolescent Psychiatry and Mental Health 7: 1-8.

19. Rich P (2005) Attachment and Sexual Offending: Understanding and Applying Attachment Theory to the Treatment of Juvenile Sexual Offenders.

20. Ainsworth M (1985) Attachments across the lifespan. 61: 792-812.

21. Venta A, Shmueli-Goetz Y, Sharp C (2014) Assessing attachment in adolescence: A psychometric study of the child attachment interview. Psychological Assessment 26: 238-255.

22. Marshall W, Hudson S, Hodkinson S (1993) The importance of attachment bonds on the development of juvenile sex offending.

23. Zaremba LA, Keiley MK (2011) The mediational effect of affect regulation on the relationship between attachment and internalizing/externalizing behaviors in adolescent males who have sexually offended. Children and Youth Services Review 33: 1599-1607.

24. Bowlby J (1973) Attachment and Loss New York, NY: Basic Books

25. Shumaker DM, Deutsch RM, Brenninkmeyer L (2009) How do I connect? 
Citation: Merino C, Underwood LA, Crump Y, Williams C (2018) The Relationship between Attachment, Depression, and Anxiety in Juveniles with Sexual Behavior Problems. J Ment Disord Treat 4: 152. doi:10.4172/2471-271X.1000152

Page 9 of 9

Attachment issues in adolescence. Journal of Child Custody 6: 91-112.

26. Elgar F, Knight J, Worrall G, Sherma G (2003) Attachment characteristics and behavioral problem in rural and urban juvenile delinquents. Child Psychiatry and Human Development 34: 35-48

27. Allen J, Moore C, Kuperminc G, Bell K (1998) Attachment and adolescent psychosocial functioning. Child Development 69: 1406-1419.

28. Slade A (1999) Attachment theory and research. Implications for the theory and practice of individual psychotherapy with adults.

29. Underwood LA, Dailey FL, Merino C, Crump Y (2015) Results from a multimodal program evaluation of a four year statewide juvenile sex offender treatment and reentry program. Journal of Prison Education and Re-entry 2: 19-32.

30. Van Eijck F, Branje S, Hale W, Meeus W (2012) Longitudinal associations between perceived parent-adolescent attachment relationship quality and generalized anxiety disorder symptoms in adolescence. Journal of Abnormal Child Psychology 40: 871-883.

31. Becker J (1998) What we know about the characteristics and treatment of adolescents who have committed sexual offenses. Child Maltreatment 3: 317329.

32. Berenson D, Underwood LA (2001) A resource guide: Sex offender programming in youth correction and detention centers. Council for Juvenile Correctional Administrators (CJCA).

33. Marshall WL, Barabee HE (1990) An integrated theory of the etiology of sexual offending.

34. Seto MC, Lalumière ML (2010) A review and test of explanations through metaanalysis. Psychological Bulletin 136: 526-575.

35. West M, Rose MS, Spreng S, Sheldon-Kelle A, Adam K (1998) Adolescent attachment questionnaire: A brief assessment of attachment in adolescence. Journal of Youth and Adolescence 27: 661-673. 